\title{
High gain dual-band couple feed transparent THz antenna for satellite communications
}

\begin{abstract}
An Indium-doped tin oxide (ITO) based optically transparent aperture coupled rectangular patch antenna is resonated at 0.750 and $1.1 \mathrm{THz}$ and then its performances is analyzed. The aperture couple feed method has been used to feed the antenna. The antenna characteristics such as bandwidth and radiation properties are investigated. The proposed antennas' specifications are investigated and then compared with both gold, copper and conventional aperture coupled rectangular antenna at the desired resonant frequencies $(0.75$ and $1.1 \mathrm{THz})$. Then to improve performance of the antenna, the patch is covered by a layer of Carbon Nano Tube (CNT). The proposed transparent antenna have achieved impedance bandwidth of $38 \%$ and $19 \%$ in the band of $0.75 \mathrm{GHz}$ and $1.1 \mathrm{THz}$ respectively. The proposed antenna has a peak gain of 7.7 and $10.3 \mathrm{~dB}$ which is better than conventional rectangular patch antenna gain; besides, the radiation efficiency is more than $85 \%$ across the operation frequency band. The design technique was verified through the simulation and the results show its capability to improve overall performance of the $\mathrm{THz}$ antennas.
\end{abstract}

Keyword: Transparent; Dual bands; Shrunk size; Loss; Performance 